\title{
Introducing Cinematic Rendering: A Novel Technique for Post-Processing Medical Imaging Data
}

\author{
Franz A. Fellner ${ }^{1,2}$ \\ ${ }^{1}$ Institute of Radiology, Kepler University Clinic, Medical Faculty of the Johannes Kepler University, Linz, Austria \\ ${ }^{2}$ Medical Faculty of the Friedrich-Alexander University of Erlangen-Nürnberg, Erlangen, Germany \\ Email:franz.fellner@akh.linz.at
}

Received 1 March 2016; accepted 26 March 2016; published 29 March 2016

Copyright ( 2016 by author and Scientific Research Publishing Inc.

This work is licensed under the Creative Commons Attribution International License (CC BY).

http://creativecommons.org/licenses/by/4.0/

(c) (i) Open Access

\begin{abstract}
Since the 1980s, various techniques have been used in the field of medicine for the post-processing of medical imaging data from computed tomography (CT) and magnetic resonance (MR). They include multiplanar reformations (MPR), maximum intensity projection (MIP) and Volume Rendering (VR). This paper presents the prototype of a new means of post-processing radiological examinations such as CT and MR, a technique that, for the first time, provides photorealistic visualizations of the human body. This new procedure was inspired by the quality of images achieved by animation software such as programs used in the entertainment industry, particularly to produce animated films. Thus, the name: Cinematic Rendering. It is already foreseeable that this new method of depiction will quickly be incorporated into the set of instruments employed in so-called virtual anatomy (teaching anatomy through the use of radiological depictions of the human body via X-ray, CT and MR in addition to the use of computer animation programs designed especially for human anatomy). Its potential for medical applications will have to be evaluated by future scientific investigations.
\end{abstract}

\section{Keywords}

Cinematic Rendering, Volume Rendering, Virtual Anatomy, Computed Tomography (CT), Magnetic Resonance (MR)

\section{Introduction}

In the field of radiology, various methods are employed for the post-processing of the results of imaging proce- 
dures such as computed tomography (CT) and magnetic resonance (MR) imaging data sets: multiplanar reformations (MPR), maximum intensity projection (MIP) and Volume Rendering (VR).

During a maximum intensity projection, rays are cast through the 3-D image data volumes and the respective data point with the maximum intensity along the direction of viewing (projection direction) is selected and depicted. Areas of application of MIP include depictions of blood vessels-i.e. CT and MR angiography data sets. MIP was originally developed for clinical use in the field of nuclear medicine; accordingly, the first version was named maximum activity projection (MAP) [1].

The earliest implementation of a Volume Rendering technique was the outcome of research conducted at the Mayo Clinic in the 1970s [2]. In the mid-80s, advances in image processing hardware and the integration of new data manipulation techniques led to the simultaneous development of Volume Rendering at the University of North Carolina [3] and at Pixar in San Rafael [4], whereby the latter had originally been an upshot of work by computer specialists in the Computer Graphics Group at Lucasfilm [2]. The work at Pixar culminated in collaboration with the CT research group at Johns Hopkins Hospital [5] [6]. Today, Volume Rendering is an established post-processing technique for 3-D CT and MR data sets, one used routinely in clinical practice.

To do so-called Cinematic Rendering, engineers developed a software prototype that can generate photorealistic depictions of the human body from CT and MR data sets and achieve results that far surpass those of previously available post-processing methods (such as Maximum Intensity Projection, Surface Shaded Display or Volume Rendering). In recognition of the fact that this technique was inspired by the quality of computer animation programs such as those utilized in the entertainment industry and especially to produce big-screen films, the developers named it Cinematic Rendering.

\section{The Software}

Siemens Healthcare, Medical Imaging Technologies, Princeton, USA recently developed a prototype of new image processing software for radiological data sets that they call Cinematic Rendering. At our institute, we have installed this software on a workstation for scientific purposes (syngo.via Frontier). This new technique delivers a considerably more photorealistic depiction of CT and MR data sets than was possible heretofore with Volume Rendering. Whereas in the case of Volume Rendering, simple ray casting [7]-[9] is performed to compute the images, Cinematic Rendering takes into account the complex interaction of photons with the human anatomy and thus generates highly photorealistic images with an unprecedented degree of realism (Figure 1). The use of clip planes makes it possible — just as in the case of Volume Rendering — to cut into the volume.

Conventional Volume Rendering techniques employ predefined color, turbidity and brightness values, whereby they proceed under the assumption of an artificial light source. Cinematic Rendering performs a physical simulation of the light diffusion, as a result of which effects such as environmental influences, shadowing, refraction, occlusion, dispersion and soft shadows attain a high dynamic range. To achieve this, a so-called spherical panorama is recorded —-for example, with a reflecting ball—which registers the actual lighting conditions in

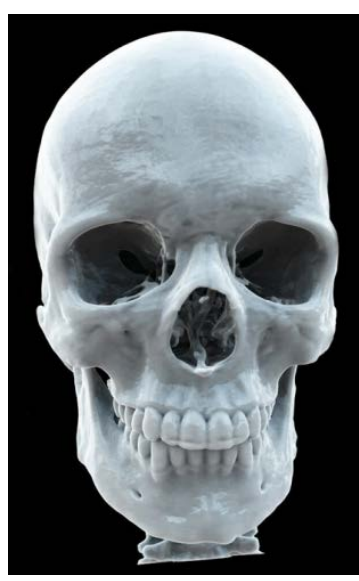

(a)

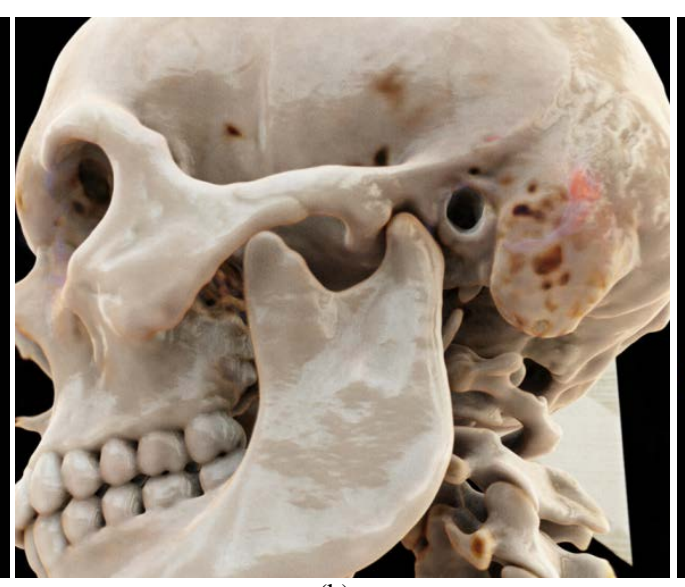

(b)

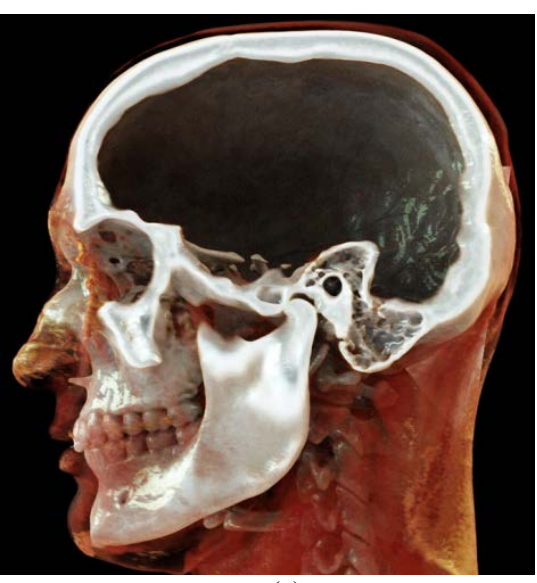

(c)

Figure 1. Cinematic Rendering of a non-contrast-enhanced CT head scan with rendering of the bony surface (a) (b) and depiction of the interior of the skull in the area of the midline by means of sagittal clip plane (c). 
order to apply them to all synthetic elements added later. In comparison to simple ray casting based Volume Rendering, Cinematic Rendering is based on the Monte-Carlo path tracing of volumetric data [10]-[12]. These and other techniques such as variable aperture width and motion blur are also utilized in the film industry. Accordingly, Cinematic Rendering was the name chosen for this new technique.

\section{Discussion}

Cinematic Rendering makes it possible to achieve higher quality three-dimensional depiction of CT and MR data sets. Due to the considerably more elaborate and thus truer-to-life simulation of the paths of light rays as employed in the animation industry, this new technique produces photorealistic images the likes of which were impossible before with Volume Rendering. Figure 2 shows two visualizations of the musculature of the thorax and abdomen derived from the same CT data set: one with Volume Rendering (a), and the other with Cinematic Rendering (b).

Anatomical structures other than the musculature (e.g. soft tissue, cartilage, bones and blood vessels) are also depicted much more photo-realistically with Cinematic Rendering than was previously possible with Volume Rendering (Figure 3).

Figure 3 is a comparative depiction of Volume Rendering (a) and Cinematic Rendering (b). Both display bony structures and blood vessels, as well as the kidneys that also show up due to the extent of the blood flow to them. Here too, the greater photorealism of Cinematic Rendering delivers a substantially better depiction of the above-named structures than Volume Rendering does.

In the Volume Rendering image, a thoracic aorta graft is hardly recognizable, whereas it is very easy to make out in the depiction produced by Cinematic Rendering (arrow in Figure 3).

It is already obvious that this new technique that delivers photorealistic depictions of the human anatomy will launch a quantum leap in quality in so-called virtual anatomy, as shown below in Figure 4.

For several years now, anatomy instruction has made use of, in addition to conventional teaching involving human cadavers, radiological scans (as obtained with x-ray, computed tomography, magnetic resonance, catheter angiography) as well as images produced by computer animation software packages specifically programmed

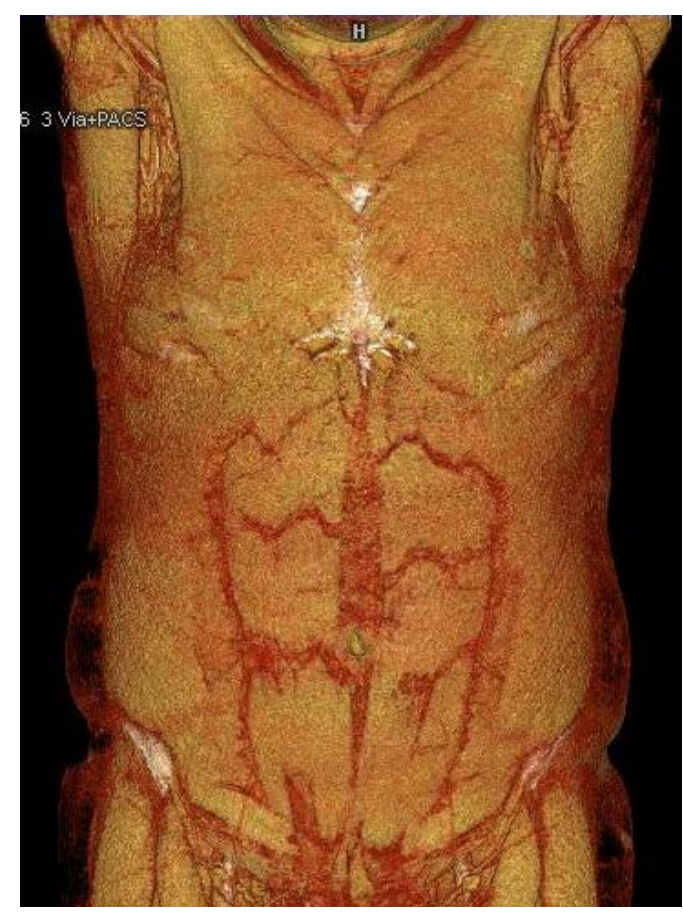

(a)

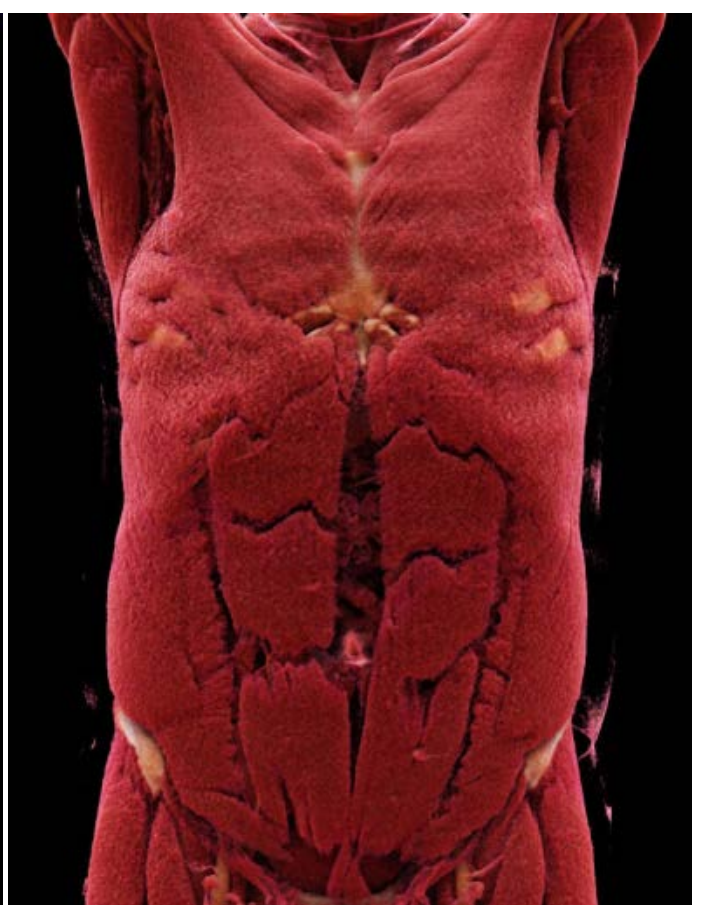

(b)

Figure 2. Volume Rendering (a) and Cinematic Rendering (b) of a whole-body CT scan with depiction of the thoracic and abdominal musculature; the musculature is depicted considerably more realistically by Cinematic Rendering than by Volume Rendering. 


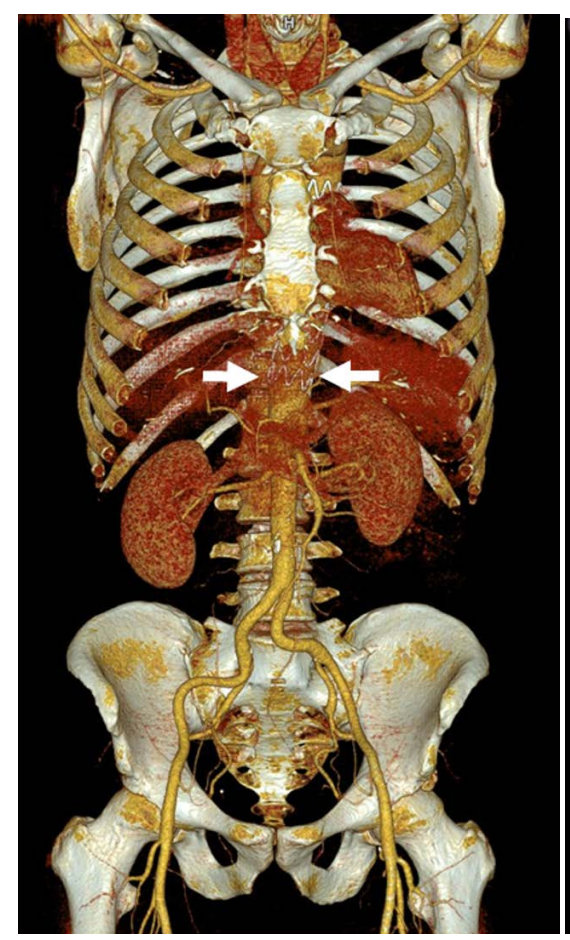

(a)

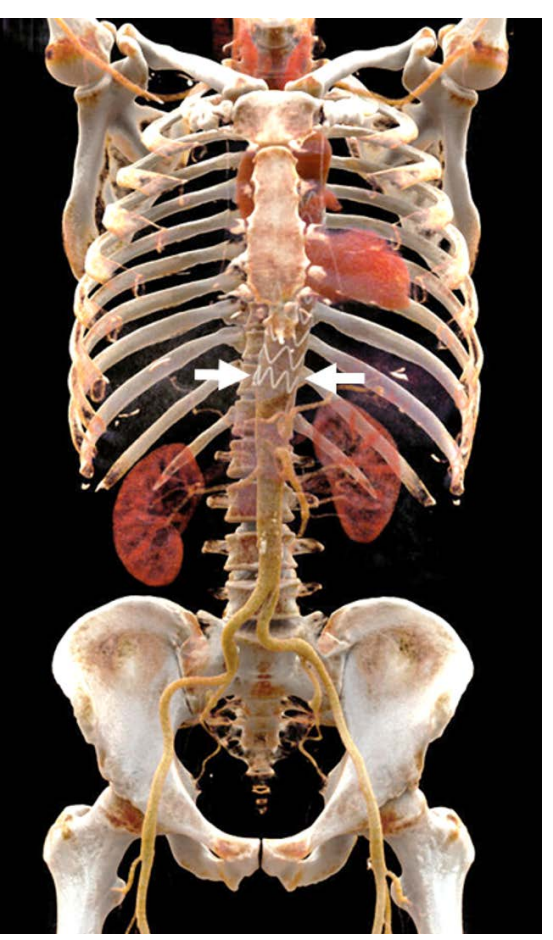

(b)

Figure 3. The same whole-body CT scan as in Figure 2; in this case, the bones, blood vessels and kidneys are visualized, and once again, a significantly more realistic depiction of the above-named anatomical structures is provided by Cinematic Rendering (b) than by Volume Rendering (a). The thoracic aorta graft (arrows) is much more clearly apparent in the Cinematic Rendering image (b).

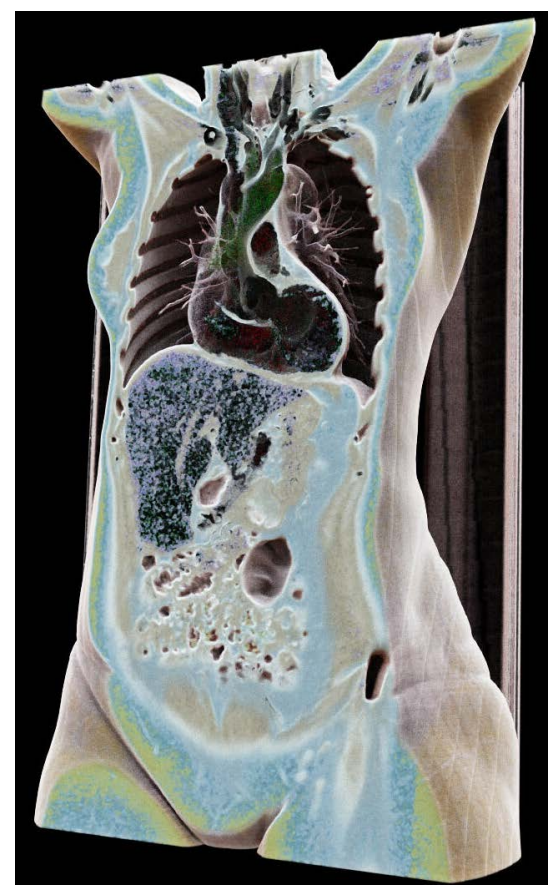

Figure 4. This Cinematic Rendering, as obtained with a coronal clip plane view, of a whole-body CT scan features an impressive depiction of the thorax with ribs including the heart and major blood vessels (aorta, pulmonary trunk and branches), as well as the liver in the right upper abdomen and sections of the small and large intestines in the abdominal cavity. 
for human anatomy that are already commercially available.

These teaching methods are referred to as virtual anatomy to differentiate them from so-called classical anatomy instruction utilizing human cadavers.

The extent to which there emerge new aspects conducive to further potential applications in the field of medicine will have to be evaluated by scientific investigations performed over the next few years.

It would seem to be feasible to apply Cinematic Rendering in the musculoskeletal sector (maxillofacial surgery, trauma surgery, orthopedic medicine) for preoperative three-dimensional examination of fractures and malpositions. This new form of three-dimensional depiction could also be of interest in other surgical disciplines such as abdominal surgery, vascular surgery, gynecology and urology (Figure 5).

As is also the case with the various Volume Rendering programs for medical applications, this Cinematic Rendering prototype features diverse templates with various color combinations and illumination options.

Templates that visualize bones especially well were used for Figure 1. The templates that were used for Figure 2, Figure 4 and Figure 5 are particularly well-suited for the depiction of soft tissue. To generate Figure 3, a palette was used that produces high contrasts and thus does an outstanding job visualizing bones as well as blood vessels filled with a contrast medium.

Already now we use routinely Cinematic Rendering for teaching human anatomy on the base of CT and MR 3D data sets (virtual anatomy), because Cinematic Rendering is able to create photorealistic depictions of the human body from CT and MR data sets achieving results that far surpass those of previously available post-processing methods, such as Maximum Intensity Projection or Volume Rendering.

Other possible applications in medicine have to be evaluated in upcoming studies, as mentioned above. At the moment this prototype is not intended for clinical use. Furthermore, even though the rendering is fully interactive, at that time the rendering of the final image needs some seconds time (5 - 30 seconds depending on the quality of the resulting image). The next step to refine this prototype will be to accelerate the rendering speed to real time high-end quality reconstruction.

\section{Conclusions}

Cinematic Rendering is a new technique for the 3-D visualization of radiological imaging data such as those provided by computed tomography and magnetic resonance imaging. This new technique now makes it possible to produce photorealistic depictions on the basis of computed tomography and magnetic resonance cross-sectional scans, which is not even close to being feasible heretofore with any of the established post-processing techniques.

This has the potential to usher in a new era in the visualization of medical imaging data provided by computed

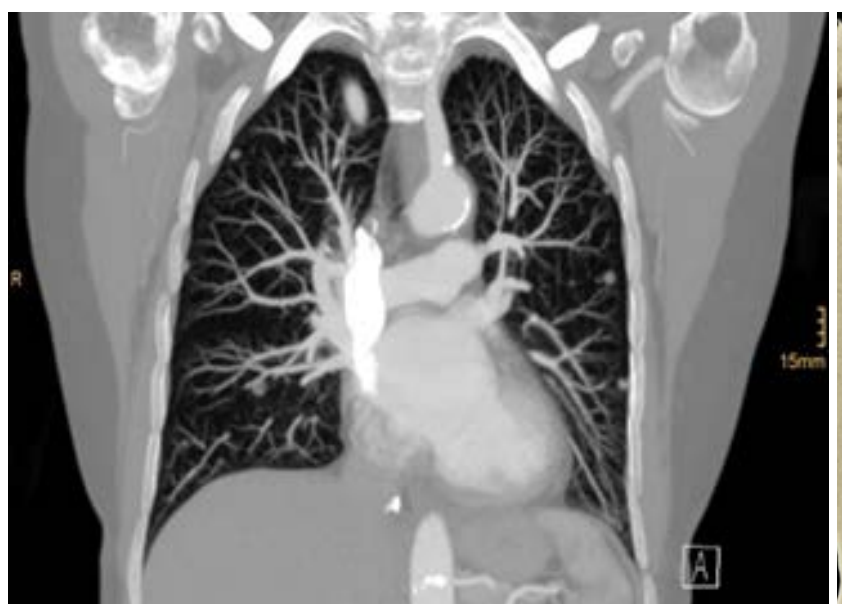

(a)

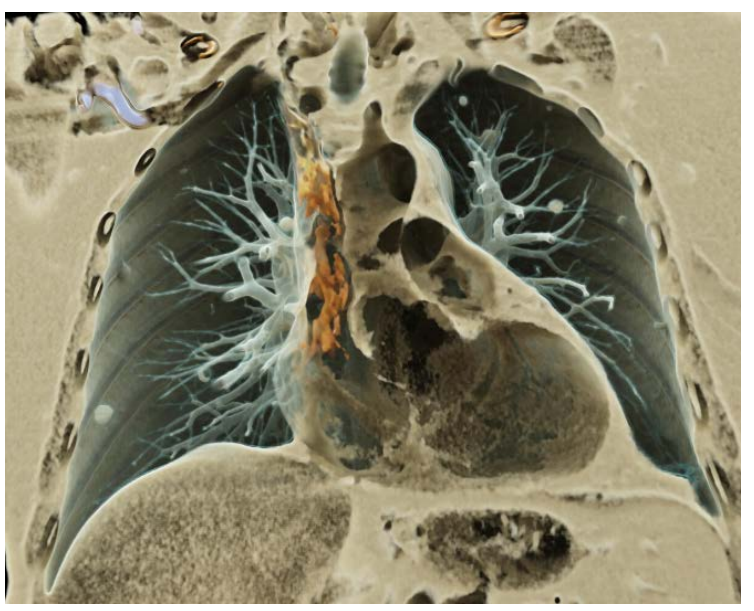

(b)

Figure 5. Comparison of Maximum Intensity Projection (MIP) (a) and Cinematic Rendering (b) of a contrast-enhanced computed tomography scan of the chest visualizing multiple pulmonary nodules. Cinematic Rendering is providing an excellent visualization of the three-dimensional topography of the thoracic wall, the mediastinum with heart and major vessels and the different multiple nodules significantly superior to MIP. 
tomography and magnetic resonance imaging.

\section{References}

[1] Wallis, J.W., Miller, T.R., Lerner, C.A. and Kleerup, E.C. (1989) Three-Dimensional Display in Nuclear Medicine. IEEE Transactions on Medical Imaging, 8, 297-303. http://dx.doi.org/10.1109/42.41482

[2] Calhoun, P.S., Kuszyk, B.S., Heath, D.G., Carley, J.C. and Fishman, E.K. (1999) Three-Dimensional Volume Rendering of Spiral CT Data: Theory and Method. Radiographics, 19, 745-764. http://dx.doi.org/10.1148/radiographics.19.3.g99ma14745

[3] Levoy, M. (1988) Display of Surfaces from Volume Data. IEEE Computer Graphics and Applications, 8, $29-37$. http://dx.doi.org/10.1109/38.511

[4] Drebin, R.A., Carpenter, L. and Hanrahan, P. (1988) Volume Rendering. Computers \& Graphics, 22, 65-74. http://dx.doi.org/10.1145/378456.378484

[5] Fishman, E.K., Drebin, B., Magid, D., Scott Jr., W.W., Ney, D.R., Brooker Jr., A.F., Riley Jr., L.H., St Ville, J.A., Zerhouni, E.A. and Siegelman, S.S. (1987) Volumetric Rendering Techniques: Applications for Three-Dimensional Imaging of the Hip. Radiology, 163, 733-738. http://dx.doi.org/10.1148/radiology.163.3.3575725

[6] Catmull, E., Fishman, E.K., Horton, K.M. and Raman, S.P. (2015) From Toy Story to CT Scans: Lessons from Pixar for Radiology. Journal of the American College of Radiology, 12, 978-979. http://dx.doi.org/10.1016/j.jacr.2014.08.010

[7] Levoy, M. (1990) Efficient Ray Tracing of Volume Data. ACM Transactions on Graphics, 9, 245-261. http://dx.doi.org/10.1145/78964.78965

[8] Westermann, R. and Ertl, T. (1998) Efficiently Using Graphics Hardware in Volume Rendering Applications. SIGGRAPH 98 Proceedings of the $25^{\text {th }}$ Annual Conference on Computer Graphics and Interactive Techniques, 169-177. http://dx.doi.org/10.1145/280814.280860

[9] Roettger, S., Guthe, S., Weiskopf, D. and Ertl, T. (2003) Smart Hardware-Accelerated Volume Rendering. Proceedings of EG/IEEEE TCVG Symposium on Visualization VisSym'03, 231-238

[10] Csébfalvi, B. and Szirmay-Kalos, S. (2003) Monte Carlo Volume Rendering. VIS'03 Proceedings of the 14th IEEE Visualization, 59. http://dx.doi.org/10.1109/visual.2003.1250406

[11] Ropinski, T., Döring, C. and Rezk-Salama, C. (2010) Interactive Volumetric Lighting Simulating Scattering and Shadowing. Proceedings of IEEE Pacific Visualization, 169-176.

[12] Kroes, T., Post, F.H. and Botha, C.P. (2012) Exposure Render: An Interactive Photo-Realistic Volume Rendering Framework. PLoS ONE, 7, e38586. http://dx.doi.org/10.1371/journal.pone.0038586 